\title{
Fatores que influenciam a hipertensão arterial sistêmica e qualidade de vida em professores universitários
}

\author{
Factors influencing systemic arterial hypertension \\ and quality of life in university teachers \\ Factores que influencen la hipertensión arterial sistémica \\ y calidad de vida en profesores universitarios \\ Aline de Paula DEVECHIO ${ }^{1}$ \\ Tamires Aparecida PELEGRINO ${ }^{1}$ \\ Giovanne Ragone Marinho da SILVA ${ }^{1}$ \\ Marcelo Studart HUNGER ${ }^{2}$ \\ Lucas Rissete DELBIM ${ }^{3}$ \\ Roberto MAGALHÃES ${ }^{4}$ \\ Sérgio Fernando ZAVARIZE ${ }^{5}$ \\ Anderson MARTELLI ${ }^{\mathbf{6}}$ \\ ${ }^{1}$ Graduando(a) em Educação Física pela Faculdade FMG - Mogi Guaçu-SP \\ ${ }^{2}$ Mestre em Performance Humana pela Universidade Metodista de Piracicaba, \\ Docente do Curso de Graduação em Educação Física da FMG - Município de Mogi Guaçu - SP \\ ${ }^{3}$ Mestre em Sustentabilidade e Qualidade de Vida - (UNIFAE), \\ Docente do Curso de Educação Física da FMG - Município de Mogi Guaçu - SP \\ ${ }^{4}$ Pós-Graduado em Fisiologia do Exercício - Universidade Federal de São Paulo, \\ Docente do Curso de Graduação em Educação Física da FMG - Município de Mogi Guaçu - SP \\ ${ }^{5}$ Doutor em Psicologia pela Pontifícia Universidade Católica de Campinas, PUC-Campinas, \\ Docente do Curso de Fisioterapia da FMG - Município de Mogi Guaçu-SP \\ ${ }^{6}$ Mestrando Ciências Biomédicas UNIARARAS, \\ Pós-Graduado em Laboratório Clínico Faculdade de Ciências Médicas, Universidade Estadual de Campinas, UNICAMP \\ Docente do Curso de Graduação em Educação Física da FMG - Faculdade FMG - Mogi Guaçu - SP
}

\begin{abstract}
Resumo
A hipertensão arterial sistêmica (HAS) é uma das doenças crônicas não transmissíveis mais comuns da atualidade, acometendo homens e mulheres. Afeta vários órgãos e raramente apresenta sinais de alerta, mas pode tornar-se hostil caso não seja tratada. Devido aos inúmeros afazeres do dia a dia e hábito de vida sedentário, a classe de professores tem sido alvo da HAS e complicações cardiovasculares. Este estudo buscou avaliar os fatores envolvidos no desenvolvimento da HAS e sua interferência na qualidade de vida de professores universitários da Faculdade Mogiana do Estado de São Paulo, município de Mogi Guaçu - SP. Participaram desta pesquisa 21 docentes dos cursos de Educação Física, Direito e Administração, sendo sete participantes de cada curso. Este estudo possibilitou maior aproximação dos fatores de riscos com a HAS junto aos docentes universitários, sendo observados fatores de riscos para o desenvolvimento da HAS na amostra pesquisada. Portanto, faz-se necessário, o incentivo de campanhas que visem à prática de atividade física regular, hoje amplamente recomendada, e a conscientização dos docentes para a adoção de um estilo de vida saudável.

Descritores: Hipertensão; Docentes; Esgotamento Profissional; Estresse Fisiológico; Qualidade de Vida.
\end{abstract}

\begin{abstract}
Systemic arterial hypertension (SAH) is one of the most common chronic noncommunicable diseases, affecting men and women. It affects several organs and rarely shows warning signs, but can become unfriendly if left untreated. Due to the numerous daily chores and sedentary living habits, the class of teachers has been the target of hypertension and cardiovascular complications. This study aimed to evaluate the factors involved in the development of hypertension and its interference in the quality of life of university professors of the Mogiana School of the State of São Paulo, Mogi Guaçu - SP. Twenty-one teachers participated in the Physical Education, Law and Administration courses, with seven participants from each course. This study made possible a closer approximation of risk factors with SAH among university teachers, and risk factors for the development of hypertension were observed in the sample studied. Therefore, it is necessary to encourage campaigns aimed at the practice of regular physical activity, now widely recommended and the awareness of teachers to adopt a healthier lifestyle. Descriptors: Hypertension; Faculty; Burnout, Professional; Stress, Physiological; Quality of Life.

\section{Resumen}

La hipertensión arterial sistémica (HAS) es una de las enfermedades crónicas no transmisibles más comunes de la actualidad, afectando a hombres y mujeres. Afecta a varios órganos y rara vez presenta señales de alerta, pero puede llegar a ser hostil si no se trata. Debido a los innumerables quehacer del día a día y hábito de vida sedentario, la clase de profesores ha sido objeto de la HAS y complicaciones cardiovasculares. Este estudio buscó evaluar los factores involucrados en el desarrollo de la HAS y su interferencia en la calidad de vida de profesores universitarios de la Facultad Mogiana del Estado de São Paulo, municipio de Mogi Guaçu - SP. Participaron de esta investigación 21 docentes de los cursos de Educación Física, Derecho y Administración, siendo siete participantes de cada curso. Este estudio posibilitó una mayor aproximación de los factores de riesgo con la HAS junto a los docentes universitarios, siendo observado factores de riesgo para el desarrollo de la HAS en la muestra investigada. Por lo tanto, se hace necesario, el incentivo de campañas que apunte a la práctica de actividad física regular, hoy ampliamente recomendada y la concientización de los docentes para la adopción de un estilo de vida saludable. Descriptores: Hipertensión; Docentes; Agotamiento Profesional; Estrés Fisiológico; Calidad de Vida.
\end{abstract}

\section{INTRODUÇÃO}

Nos dias atuais, as condições de vida urbana contribuem significativamente para o surgimento de doenças crônicas não transmissíveis (DCNTs) e fazem das doenças cardiovasculares um problema da modernidade ${ }^{1}$. Dentre as
DCNTs, temos a hipertensão arterial sistêmica (HAS), a qual apresenta uma alta incidência na população mundial e sua etiologia é multifatorial, envolvendo fatores genéticos, ambientais e psicológicos ${ }^{2,3}$. No Brasil, as doenças 
cardiovasculares são responsáveis por mais de 250.000 mortes por ano, e a HAS participa de quase metade delas ${ }^{4}$.

A HAS se destaca por apresentar uma historia natural prolongada, multiplicidade de fatores de risco, integração de causas etiológicas e biológicas bem estabelecidas e outras ainda desconhecidas, marcada por longo período de latência apresentando curso clínico em geral assintomático e constante para toda a vida, com períodos de manifestações clínicas estáveis e outros de exacerbação, evoluindo para graus variados de incapacidades ou mesmo à morte .

A HAS possui duas classificações: a hipertensão primária, também denominada de essencial ou idiopática, representando $90 \%$ dos casos e cuja etiologia é desconhecida e a hipertensão secundária, associada a uma entidade patológica $^{6,7}$ como a hipertensão renovascular, causada por uma estenose de artéria renal, sendo a causa mais comum de HAS secundária, respondendo por $5 \%$ de todos os $\operatorname{casos}^{8,9}$. Neste sentido, uma pesquisa realizada por Martelli ${ }^{10}$ descreve a interação íntima e complexa entre o rim e a pressão arterial (PA), sendo observado que a maioria dos pacientes com algum tipo de doença renal desenvolve ou agrava os quadros de HAS.

Dentre as etiologias da HAS essencial, temos atualmente a obesidade, envelhecimento, diabetes mellitus e a insuficiência renal crônica como importantes fatores associados à dificuldade de controlar a $\mathrm{PA}^{6,11}$, podendo fazer parte desse grupo um conjunto de fatores de risco associado ao metabolismo dos lípides ${ }^{12}$ e a ingestão aumentada de cloreto de sódio ${ }^{13}$

De acordo com a VI Diretrizes Brasileiras de Hipertensão ${ }^{14}$, a mortalidade por doença cardiovascular (DCV) aumenta progressivamente com a elevação da PA a partir de 115/75 $\mathrm{mmHg}$ de forma linear, contínua e independente. No Brasil, Miranzi et al. ${ }^{15}$ salientam que 17,6\% das internações são em virtude da HAS, e que são gastos com esse agravo 5,9\% dos recursos despendidos pelo Sistema Único de Saúde (SUS).

Quanto ao diagnóstico da HAS, a medida da PA é o método mais recomendado pela Sociedade Brasileira de Hipertensão Arterial e deve ser realizada em toda consulta e/ou avaliação de saúde ${ }^{16}$. Uma única medição acima de 140/90mmHg indica que a PA está alta naquele momento, mas não é suficiente para diagnosticar a HAS, sendo necessário repetir a medição em diferentes circunstâncias e durante um determinado período de tempo que o seu médico determinará em cada caso analisado ${ }^{16}$.

Mesmo evidenciando que a HAS constitui um dos principais problemas de saúde, o número de hipertensos tratados é pequeno. Cerca de $50 \%$ desconhece sua condição e $50 \%$ da parcela que detém o conhecimento sobre seu quadro não se trata ${ }^{17}$. Existem evidências suficientes sobre o benefício do tratamento precoce da HAS, o qual pode ser baseado em quatro medidas: não farmacológicas ou mudanças no estilo de vida; remoção da causa, quando for identificada; farmacológica com adesão ao tratamento e por fim, a associação desse conjunto de medidas ${ }^{18}$.

Carvalho et al. ${ }^{19}$ demonstraram em seu estudo que, os pacientes hipertensos apresentam uma diminuição significativa da qualidade de vida relacionada à saúde quando comparados com os normotensos, corroborando com outros resultados. Pinotti et al. ${ }^{20}$ relatam que a HAS interferiu de maneira significativa na qualidade de vida na amostra estudada, principalmente em relação ao trabalho, hábitos de vida e devido ao cuidado contínuo, decorrente do caráter crônico da doença, descrevendo que essa interferência ocorreu, sobretudo, nos hábitos de vida, como o cuidado com a alimentação e a necessidade de ingerir medicamentos todos os dias, sem os quais referem sentir-se mal.

Em referência à atuação do professor universitário, Carloto e Camara ${ }^{21}$ retratam que esse profissional deve conciliar atividades de ensino, pesquisa e extensão, atendendo à produção científica das instituições, além de ter que executar funções administrativas. Franco ${ }^{22}$ descreve em seu estudo que este profissional está permanentemente sob crivo crítico, desde o ingresso na carreira, sendo avaliado em todo momento, da submissão de trabalhos em eventos, apresentação de projetos e relatórios de atividades e pesquisa, expondo a fatores estressantes que, se persistentes, podem levar ao desenvolvimento de inúmeras doenças, dentre elas a HAS.

Santos e Marques $^{23}$ realizaram um estudo em professores da educação básica e encontraram uma prevalência de HAS em 20,3\% dos avaliados. Moreira et $\mathrm{al}^{24}$ analisando professores do ensino superior, foi encontrada associação da HAS com importantes fatores de risco cardiovascular. Estes estudos ressaltam a necessidade da avaliação do estado de saúde destes profissionais, pois os mesmos possuem um papel importante na formação dos jovens, assim, o objetivo desse estudo foi identificar os principais fatores envolvidos no desenvolvimento da HAS e sua influência na qualidade de vida em professores universitários de cursos distintos - Educação Física (ED FIS), Administração (ADM) e Direito (DIR) da Faculdade Mogi Guaçu, FMG, localizada no município de Mogi Guaçu-SP.

\section{MATERIAL E MÉTODO}

Trata-se de uma investigação de caráter quantitativo, transversal, observacional e descritivo, realizado seguindo as normas que regulamentam a pesquisa em seres humanos, contidas na Resolução n ${ }^{\circ}$ 466/12 do Conselho Nacional de Saúde, aprovado pelo Comitê de Ética e Pesquisa da Faculdade Municipal Professor Franco Montoro, tendo recebido parecer favorável sob o registro CEP/FMPFM: 1.813.713/16.

A amostra foi composta por 21 professores universitários de ambos os sexos, escolhidos de forma aleatória, regularmente registrados e lecionando nos cursos de graduação em ED FIS, ADM e DIR da Faculdade FMG, sendo 7 docentes de cada curso. O recrutamento dos professores foi realizado antes do início das aulas na Sala dos Professores. Após os esclarecimentos sobre os objetivos e a metodologia da pesquisa, os que concordaram em participar da pesquisa, assinaram o Termo de Consentimento Livre e Esclarecido (TCLE) conforme preconizado na legislação vigente.

Para a coleta de dados, foi empregado um questionário semiestruturado elaborado segundo estudo de Martelli e Zavarize, ${ }^{25}$ abordando dados pessoais, profissionais, ritmo e estilo de vida, e fatores como estresse, tabagismo, etilismo, alto teor de sódio na alimentação e sedentarismo como fatores modificáveis no desenvolvimento da HAS e da qualidade de vida. Após esta etapa, a amostra foi submetida à aferição do Índice de Massa corporal - IMC utilizando a metodologia proposta por $\operatorname{Costa}^{26}$, dividindo a massa corporal em quilogramas pelo quadrado da estatura em metros $\left(\mathrm{kg} / \mathrm{m}^{2}\right)$. O equipamento utilizado para medir a estatura dos avaliados foi um estadiômetro da marca Kawee ${ }^{\circledR}$ com precisão de $1 \mathrm{~mm}$ e para a coleta da massa corporal uma balança antropométrica da marca Toledo® com precisão de $100 \mathrm{~g}$ e capacidade máxima de $200 \mathrm{Kg}$, 
aferida e calibrada, garantindo confiabilidade para a realização de tal procedimento.

Para aferição da PA, a amostra permaneceu em repouso por 15 minutos absoluto e após esse período, procedeu à verificação da PA utilizando um esfigmomanômetro marca Missouri, modelo Aneróide do tipo mecânico e estetoscópio. As medidas de PA foram obtidas no membro superior esquerdo, na posição sentada. Quanto aos valores de PA registrados, a amostra foi classificada segundo os critérios estabelecidos pela VI Diretrizes Brasileira de Hipertensão Arterial ${ }^{14}$.

Como critérios de inclusão da amostra na pesquisa, foram analisadas informações comprobatórias sobre a situação regular de registro do docente na Instituição e estar lecionando nos cursos de ED FIS, ADM e DIR da Faculdade FMG, não fazer uso de anti-hipertensivos e o aceite formal em participar da pesquisa com a assinatura do TCLE. Como critério de exclusão, não estar lecionando regularmente nos respectivos cursos, gestantes, uso de anti-hipertensivo e pelo não consentimento em participar da pesquisa.

Após a coleta de dados, as informações foram digitadas e tabuladas em banco de dados do programa Microsoft Excel - 2010 para as análises estatísticas de frequência, média e desvio padrão. Posteriormente foi realizada uma comparação dos resultados observados com os descritos na literatura.

\section{RESULTADOS E DISCUSSÃO}

Foram pesquisados 21 professores vinculados à Faculdade FMG, localizada no município de Mogi GuaçuSP, sendo 7 professores dos respectivos cursos: ADM, DIR e ED. FIS. A amostra foi composta por 1 mulher e 6 homens da ED FIS e 3 mulheres e 4 homens da ADM e DIR respectivamente, com faixa etária entre 28 a 66 anos e média de idade e desvio padrão por curso - ADM $(37 \pm 6,61)$, DIR $(44 \pm 12,45)$ e ED FIS $(40 \pm 6,24)$. De acordo com Woods et al. $^{27}$ a prevalência da HAS aumenta com a idade, sendo maior em pessoas de etnia negra, indivíduos com menor grau de instrução e poder socioeconômico. $\mathrm{O}$ mesmo autor enfatiza que é mais frequente em homens jovens e de meia idade com uma inversão dessa tendência no idoso, tornandose mais prevalente nas mulheres.

$\mathrm{Na}$ docência do ensino superior, muitos profissionais estão vinculados a mais de uma instituição. Em relação ao vínculo empregatício, os professores de ED FIS $(n=4 ; 57 \%)$ relataram estar trabalhando de forma excessiva, na ADM e DIR, $(n=2)$, respondendo por $29 \%$, sendo referida pelos docentes uma carga horaria de trabalho de 10 a 12 horas por dia, perfazendo um fator predisponente para a redução da qualidade de vida e o desenvolvimento de comorbidades, dentre elas, as cardiovasculares.

Os dados obtidos nos presente trabalho corroboram com uma pesquisa realizada por Alves et al. ${ }^{28}$ com 30 professores universitários, sendo observado que $53 \%$ trabalham entre oito e 12 horas por dia, $40 \%$ referiram trabalhar mais que 12 horas por dia e apenas uma quantidade ínfima - 6,7\% trabalham entre cinco e oito horas por dia e de acordo com os autores, esse fator pode levá-los ao sedentarismo, estresse e privação do sono contribuindo para o aumento dos valores tensionais da PA. Neste sentido, Baião e Cunha, ${ }^{29}$ descrevem que o trabalho docente é caracterizado por uma baixa remuneração, déficit estrutural e carga horária elevada contribuindo para o desenvolvimento de muitas doenças ou disfunções ocupacionais. Araújo et al. $^{30}$ referem que esses fatores geram uma insatisfação profissional o que contribui para o estresse ocupacional e o desenvolvimento da HAS e outras DCNTs.

Esses profissionais, além de realizarem atividades de docência, realizam tarefas domésticas, a educação dos filhos e os afazeres do dia a dia somadas às tarefas relacionadas à docência executadas extraclasse como o planejamento das aulas, correção das avaliações e trabalhos, realização de pesquisas e orientações, dentre outros, acelerando muito o ritmo de vida. Assim, a amostra foi questionada sobre o ritmo de vida. Na ADM e ED FIS, 71\% dos docentes apresentam um ritmo de vida acelerado e $29 \%$ um ritmo intermediário (nem calmo ou acelerado). Os docentes do DIR, $14 \%$ calmo, $43 \%$ intermediário e $43 \%$ acelerado. Os docentes de ADM e ED FIS mostraram números preocupantes quanto ao ritmo de vida.

Uma pesquisa $^{31}$ retratou os motivos que levaram os docentes de ADM de uma instituição superior a trabalhar em dupla jornada, sendo observado que $44 \%$ da amostra ressaltaram o complemento financeiro familiar, $31 \%$ a possibilidade de desenvolvimento profissional e $25 \%$ não responderam a esse questionamento. Batista et al. ${ }^{32}$ relatam que as exigências da vida moderna fazem com que os indivíduos apresentem um ritmo de vida cada vez mais acelerado fazendo-o produtivo e rentável em todos os momentos, dissociando cada vez menos as horas trabalhadas do tempo reservado ao lazer comprometendo sua qualidade de vida.

Quando indagados sobre tempo disponível para lazer, $71 \%$ dos docentes de ED FIS e ADM ( $\mathrm{n}=5$ por curso) e $87 \%$ dos docentes de DIR $(n=6)$ relataram apresentar tempo para essa prática. Para os docentes que referiram ter um período para o lazer, na ADM 60\% $(\mathrm{n}=3)$ relataram que esse período ocorre de segunda a sexta feira e $40 \%(n=2)$ aos finais de semana; na ED FIS $40 \%$ dos docentes $(n=2)$ apontaram o período para lazer durante a semana e $60 \%(n=3)$ aos finais de semana. No DIR $17 \%(\mathrm{n}=1)$ responderam que o tempo para lazer é no decorrer da semana e $83 \%(n=5)$ aos finais de semana. Foi possível observar que grande parte dos docentes apresenta tempo disponível para essa atividade, seja ela durante a semana e/ou aos finais de semana. Para os docentes do DIR, o tempo para o lazer está praticamente restrito aos finais de semana. Fatores relacionados aos baixos salários, aquisição de dupla jornada e uma carga horária de trabalho, que pode incluir os finais de semana, podem estar relacionados à redução do lazer junto aos docentes ${ }^{33}$.

$\mathrm{Na}$ sequência, a amostra foi questionada sobre o cansaço do dia a dia provindo das atividades desenvolvidas durante a semana e muitas vezes aos finais de semana. Na ADM e ED FIS, 57\% ( $n=4 /$ curso) relataram cansaço excessivo e no DIR 43\% (n=3). Na subclassificação desse cansaço - físico, mental ou ambos, a ADM apresentou $25 \%$, $25 \%$ e $50 \%$, a ED FIS $0 \%, 25 \%$ e $75 \%$ e o DIR $0 \%, 34 \%$ e $66 \%$, respectivamente (Figura 1). Em relação ao uso de alguma substância como forma de melhorar o cansaço, dois docentes do DIR relataram fazer uso, representando $9 \%$ da amostra total.

Nos últimos anos, inúmeros estudos evidenciaram que o sono repercute diretamente em alterações cardiovasculares, dentre elas a HAS. Quanto às horas de sono por noite, foi observado que os docentes pesquisados apresentam de seis a oito horas de sono por noite - ADM e ED FIS, $57 \%(n=4)$ e DIR, $100 \%(n=7)$. Em relação à insônia, um docente da ADM relatou apresentar $-14 \%$.

De acordo com Drager e Lorenzi-Filho ${ }^{34}$ o sono é um 
período heterogêneo que participa ativamente no controle da PA, exercendo importante função durante o período da vigília. Mathias et al. ${ }^{35}$ referem o sono como um fator imprescindível para a manutenção de uma melhor qualidade de vida e sua falta pode ter uma repercussão no aprendizado, redução da motivação e concentração, déficit de memória, alterações de humor entre outros.

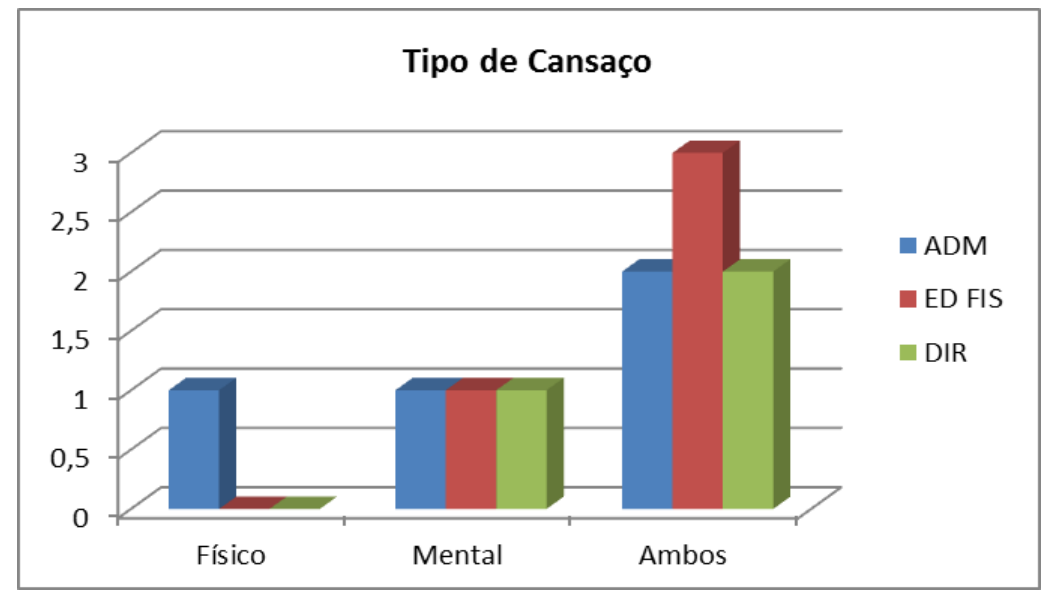

Figura 1: Distribuição da amostra por tipo de cansaço.

Nesta profissão, acaba sendo um hábito comum a execução de trabalho em casa como usar o computador para pesquisa, preparação de aulas e provas, favorecendo um atraso no horário de dormir ou uma má qualidade de sono. A manutenção deste estilo de vida pode predispor esse profissional à obesidade ${ }^{36}$, diabetes e alterações cardiovasculares ${ }^{37}$.

Hábitos alimentares inadequados é outro fator preponderante no desenvolvimento de DCNTs, dentre elas a HAS $^{9}$. Nesse estudo, foi verificado que $14 \%$ dos docentes da ADM e ED FIS não se alimentam de forma regular. Neste contexto, Brown et al. ${ }^{38}$ retratam que o consumo de sódio através da alimentação em inúmeros países tem sido excessivo, variando de 9 a 12 g por pessoa/ dia. O Brasil está entre os maiores consumidores de sal, com média de ingestão de 15,09 gramas diários ${ }^{39}$.

$\mathrm{Na}$ análise da amostra quanto à ingestão de um alto teor de cloreto de sódio (sal) na alimentação, foi observada que os docentes da ADM e DIR, $(n=2), 29 \%$ e na ED FIS, $(n=1), 14 \%$, relataram ingerir grandes quantidades de cloreto de sódio na alimentação, (Figura 2). Está bem estabelecida na literatura a associação do consumo excessivo de cloreto de sódio e o desenvolvimento das DCNTs como a HAS, doenças renais e outras patologias ${ }^{28,40}$. Alves et al ${ }^{28}$ em uma pesquisa com 30 docentes, foi observado que $90 \%$ referem ingerir pouco sal na dieta e $10 \%$ consomem muito sal corroborando com os dados observados.

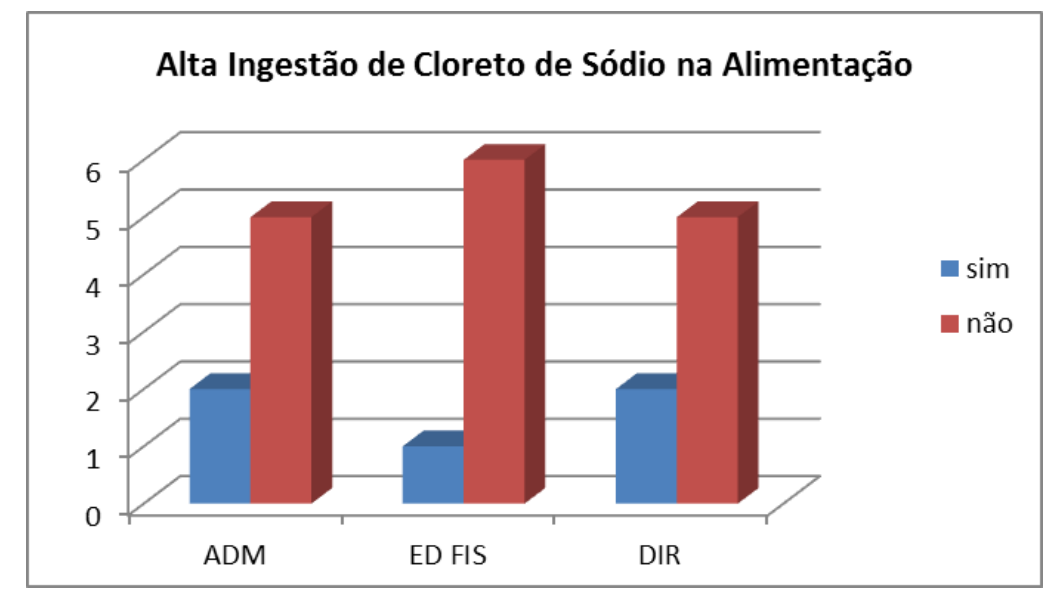

Figura 2: Ingestão de cloreto de sódio nas refeições por curso.

Outros fatores de riscos para HAS pesquisados foram o uso de tabaco e etílicos. Fazendo a utilização de tabaco, foi observado um docente da ADM - 14\%. Quanto ao uso de etílicos, dois docentes da ADM, (29\%), quatro da ED FIS,
(57\%) e três do DIR (42\%), relataram fazer uso dessa substância de forma esporádica (Figura 3).

Os resultados retratados por Alves et al. ${ }^{28}$ vão de encontro ao observado neste estudo com docentes do ensino superior, onde $13,3 \%$ de sua amostra afirmaram fazer uso do tabaco e $40 \%$ retrataram consumir algum tipo de bebida alcoólica. A V Diretrizes Brasileiras de Hipertensão Arterial $^{41}$ refere que o consumo elevado de bebidas alcoólicas favorecem o aumento da PA, podendo variar de acordo com o gênero, quantidade ingerida e sua frequência. Foi verificado a redução média de $3,3 \mathrm{mmHg}$ na PA sistólica e 2,0 mmHg na PA diastólica com a redução no consumo de etanílicos ${ }^{41}$.

Quanto ao estilo de vida e controle da HAS, o sedentarismo está como um dos principais fatores modificáveis. O exercício físico regular mostra-se como uma estratégia eficaz na redução de enfermidades clínicas decorrentes da $\mathrm{HAS}^{42}$. Na amostra, foi verificado que uma parcela significativa dos docentes realizam algum tipo de atividade física durante a semana, sendo $71 \%$ dos docentes da ADM e $86 \%$ do DIR e ED FIS respectivamente (Figura 4). Segundo Matavelli et al. ${ }^{7}$ a prática regular de atividades físicas é fundamental nas condutas não medicamentosas de prevenção e tratamento da HAS e outras patologias do sistema cardiovascular.

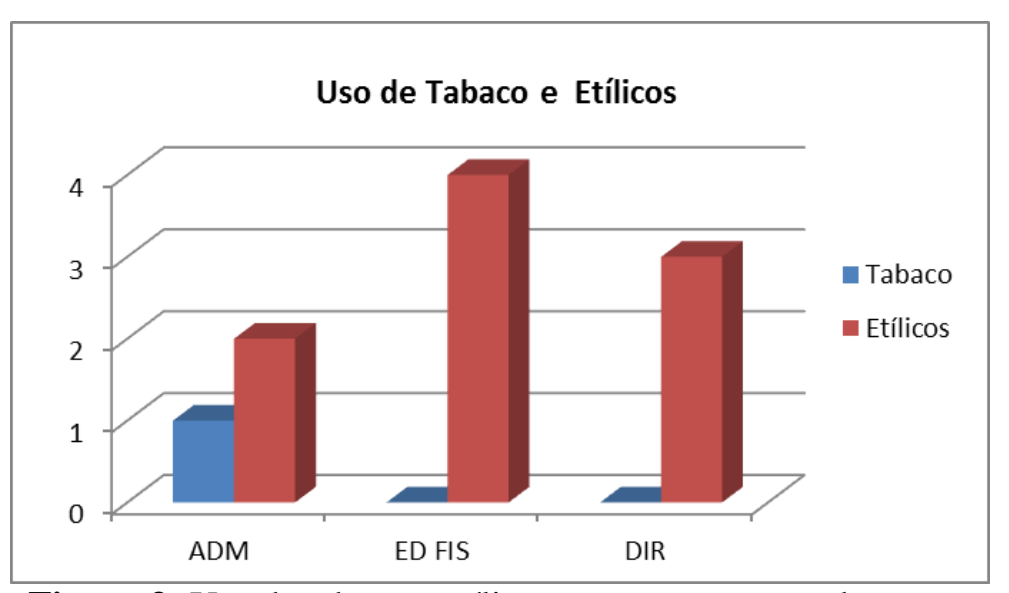

Figura 3: Uso de tabaco e etílico por curso entre os docentes.

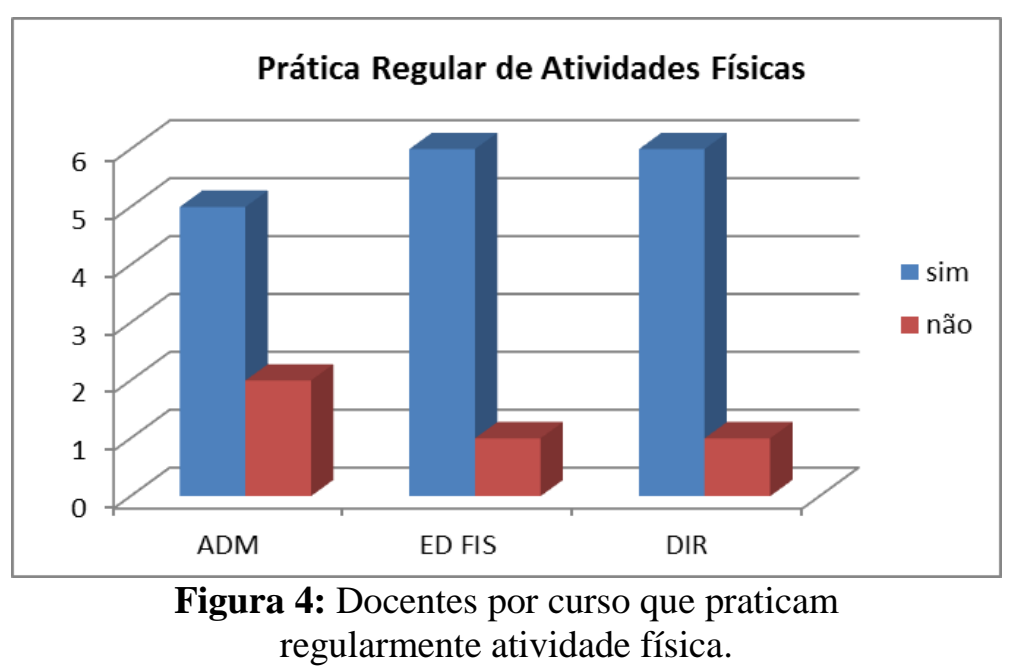

Bezerra et al. $^{43}$ retratam que a prática de exercício físico bem orientado e levando em consideração o perfil individual de cada pessoa, apresenta efeito positivo no tratamento da HAS. Neste sentido, Halbert et al. ${ }^{44}$ apontam que o treinamento físico aeróbio reduziu a PA sistólica em 4,7 $\mathrm{mmHg}$ e a PA diastólica em 3,1 $\mathrm{mmHg}$, quando comparadas ao grupo controle sem treinamento físico. Matavelli et al. $^{7}$ enfatizam em seu estudo ser primordial a prática de atividades físicas para que as pessoas tenham um futuro com mais qualidade de vida.

$\mathrm{O}$ excesso de peso e a vida sedentária aparecem como um duplo fator de prevalência para o desenvolvimento da HAS. Neste sentido, foi verificado o IMC dos docentes dos 
respectivos cursos, sendo observados números preocupantes. $\mathrm{Na}$ ADM e DIR, 29\% da amostra $(\mathrm{n}=2)$ e na ED FIS, $71 \%$ $(\mathrm{n}=5)$ apresentaram sobrepeso. Classificado como obesidade grau I, um docente da ADM e um da ED FIS, representando $14 \%$ de cada curso e na classificação de obesidade grau II e grau III, um docente do DIR e um da ADM respectivamente (Figura 5). É possível observar uma parcela significativa de professores do curso de ED FIS classificados como sobrepeso, porém, Oliveira et al. ${ }^{45}$ retratam que o IMC, apesar de ser mundialmente aceito como um referencial para estabelecer níveis de sobrepeso e obesidade, apresenta uma vulnerabilidade não considerando a massa corporal magra, uma questão a ser levada em consideração nos homens, uma vez que a amostra da ED FIS foi composta por seis homens representando $86 \%$.

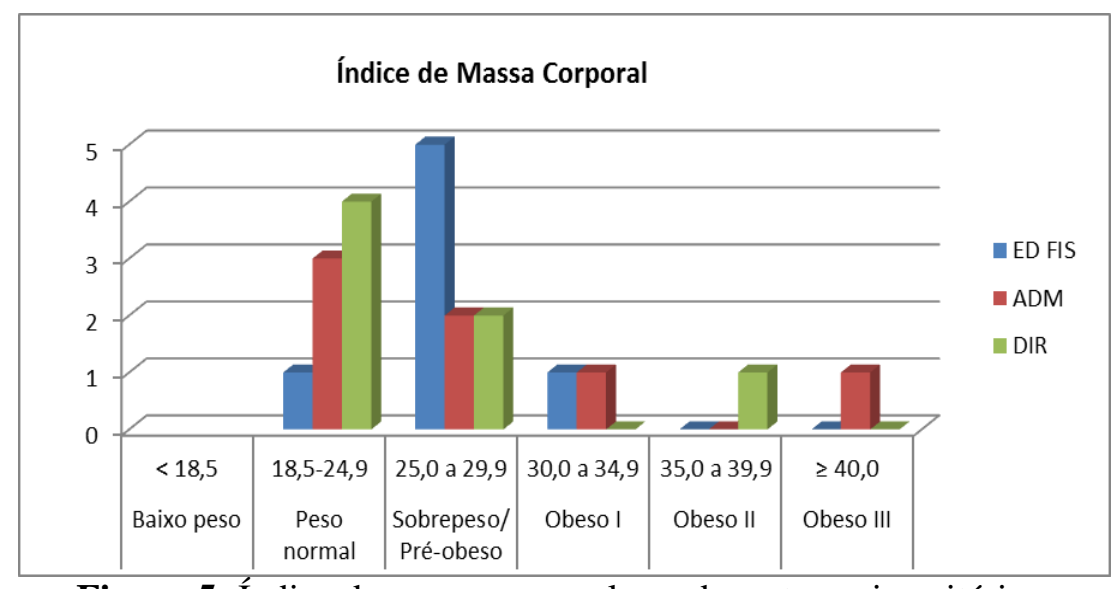

Figura 5: Índice de massa corporal nos docentes universitários.

Pesquisas em diferentes populações apontam que o sobrepeso e a obesidade podem ser responsáveis por $65 \%$ a $70 \%$ do risco de desenvolvimento de HAS ${ }^{46}$. Um estudo com 145 professores universitários foi verificado que $51,04 \%$ da amostra foram classificados com excesso de peso $^{45}$.

Dentre os fatores envolvidos na fisiopatogênese da HAS, Barreto-Filho e Krieger ${ }^{47}$ retratam que um terço deles podem ser atribuído a fatores genéticos. $\mathrm{Na}$ amostra pesquisada foi observado um histórico familiar positivo de HAS. Na ADM, 6 docentes relatam ter casos na família de HAS, representando 86\%, na ED FIS e DIR, $5-71 \%$ (Figura 6). Os resultados corroboram com um estudo de Costa et al. ${ }^{48}$, que analisaram 608 indivíduos no município de Pelotas-RS, sendo verificado que cerca de $60 \%$ dos indivíduos possuíam história familiar de doença hipertensiva.

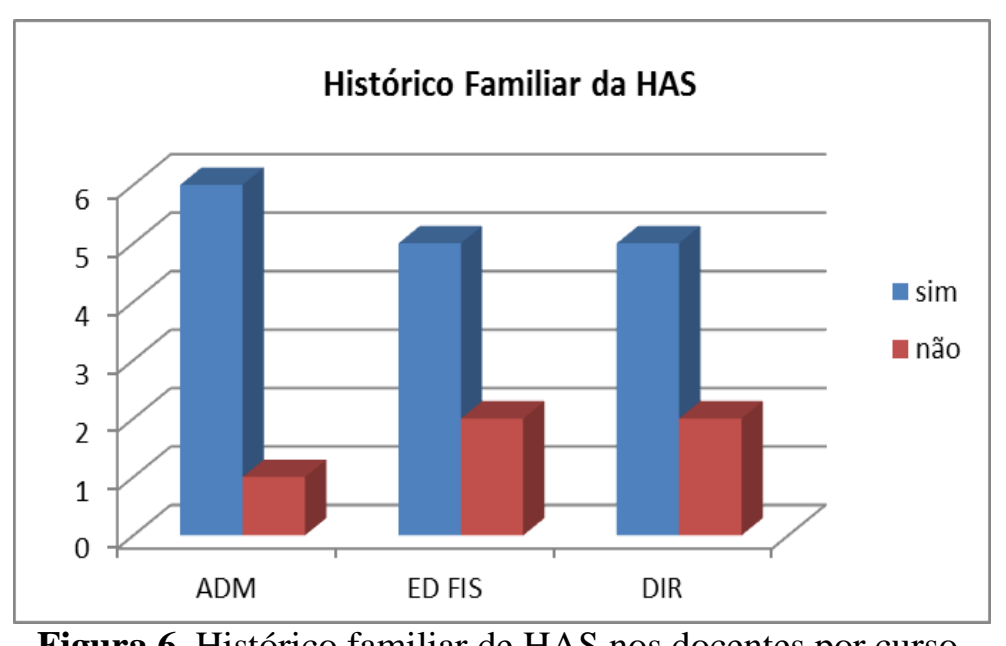

Figura 6. Histórico familiar de HAS nos docentes por curso.

Quanto aos resultados dos níveis pressóricos da amostra analisada, esta foi classificada de acordo com a VI Diretrizes Brasileiras de Hipertensão Arterial $^{14}$. Foram considerados como PA ótima $(<120<80)$, normal $(<130<$ 85), limítrofe (130-139; 85-89), hipertensão estágio 1 (140159; 90-99) e hipertensão estágio 2 (160-179; 100-109).
Em referência aos níveis pressóricos da amostra total, $66,70 \%(\mathrm{n}=14)$ estavam com a PA ótima 9.50\% ( $\mathrm{n}=2)$, pressão normal,9,50\% $(\mathrm{n}=2)$, pressão limítrofe e por fim, 14,30\% (n=3) nível 1 de hipertensão de acordo com a classificação da Sociedade Brasileira de Hipertensão (Tabela 1).

Tabela 1. Medidas da aferição da pressão arterial dos docentes

\begin{tabular}{l|c|c|c|c}
\hline \multicolumn{1}{c|}{$\begin{array}{c}\text { Classificação } \\
\text { PA sistólica e diastólica** }\end{array}$} & \multicolumn{3}{c|}{$\begin{array}{c}\text { Frequência Absoluta } \\
\text { por Curso (n) }\end{array}$} & $\begin{array}{c}\text { Frequência } \\
\text { Relativa (\%) }\end{array}$ \\
\hline & ADM & ED. Fís & DIR & \\
\hline Ótima $<120<80$ & 5 & 5 & 4 & 66,70 \\
Normal $<130<85$ & 0 & 1 & 1 & 9,50 \\
Limítrofe 130-139 85-89 & 0 & 1 & 1 & 9,50 \\
$\begin{array}{l}\text { Hipertensão estágio 1 140-159 90- } \\
\text { 99 }\end{array}$ & 2 & 0 & 1 & 14,30 \\
\hline Total & 7 & 7 & 7 & $100 \%$ \\
** VI Diretrizes Brasileiras de Hipertensão Arterial &
\end{tabular}

Docentes dos cursos de ADM e DIR apresentaram níveis elevados de HAS. Na ADM esse mesmo resultado aparece quando retratado a ingestão de alta concentração de cloreto de sódio e o uso de tabaco (Figuras 2 e 3), fatores que podem estar relacionados ao nível elevado de PA apresentado no momento da coleta.

Em estudo realizado por Silva e Souza $^{49}$ foi verificado que $10 \%$ da amostra apresentaram índices pressóricos elevados. Alves et al. ${ }^{28}$ evidenciou que 6,7\% apresentaram valores pressóricos elevados e 50\% da amostra de professores apresentaram mais de seis fatores de risco para o desenvolvimento da HAS.

Pelos resultados observados neste estudo, a atuação dessa classe profissional exige um elevado nível de escolaridade, fator que pode propiciar conhecimento sobre esta doença, redução dos fatores de riscos e a produção de conhecimento gerada nos últimos anos pelo curso de ED FIS pode estar contribuindo para a conscientização dos docentes dessa instituição como a adoção de medidas que favoreçam hábitos de vida saudáveis, melhor qualidade de vida e redução dos níveis pressóricos.

\section{CONCLUSÃO}

A HAS tem contribuído para uma elevada mortalidade cardiovascular em todo país, representando um sério problema de saúde pública. $\mathrm{O}$ presente estudo possibilitou uma maior aproximação dos fatores de riscos com a HAS junto aos docentes universitários. Os resultados demonstram a presença de diversos fatores de risco para o desenvolvimento da HAS como o trabalho excessivo e desgastante, cansaço físico e mental, alimentação com alto teor de cloreto de sódio e sobrepeso.

Portanto, faz-se necessário, o incentivo de campanhas que visem à prática de atividades físicas regulares, hoje amplamente recomendadas e a conscientização dos docentes para a adoção de estilo de vida mais saudável, uma vez que o docente do ensino superior está exposto a ambientes conflituosos e de alta exigência de trabalho.

\section{REFERÊNCIAS}

1. Teixeira ER, Lamas AR, Costa e Silva J, Matos RM. O estilo de vida do cliente com hipertensão arterial e o cuidado com a saúde. Esc Anna Nery $R$ Enferm. 2006;10(3):378-84.

2. Miranda RD, Perrotti TC, Bellinazzi VR, Nóbrega TM, Cendoroglo MS, Tomiolo Neto J. Hipertensão arterial no idoso: peculiaridades na fisiopatologia, no 
diagnostico e no tratamento. Rev Bras Hipertens. 2002; 9(3):293-300.

3. Dórea EL, Lotufo PA. Epidemiologia da hipertensão arterial sistêmica. Hipertensão 2004;7(3):86-9.

4. Santos ZMSA. Hipertensão arterial: um problema de saúde pública. Rev Bras Promoç Saúde. 2011; 24(4):285-6.

5. Brum PC. Hipertensão Arterial e exercício físico aeróbico. In: Cardiologia do Exercício: do atleta ao cardiopata. 2 ed. São Paulo: Manole; 2006.

6. Viegas C. Sal e doença cardiovascular. Factores de Risco. 2008; 10:12-8.

7. Matavelli IS, Del Judice EL, Matavelli R, Hunger MS, Martelli A. Hipertensão arterial sistêmica e a prática regular de exercícios físicos como forma de controle: Revisão de Literatura. R bras ci Saúde. 2014; 18(4):359-66.

8. Feldman A, Freitas LZF, Collet CA, Mota AR, Pimenta $\mathrm{E}$, Sousa $\mathrm{M}$ et al. A relação entre estenose de artéria renal, hipertensão arterial e insuficiência renal crônica. Rev Bras Hipertens. 2008; 15(3): 181-4.

9. Martelli A. Estenose da artéria renal e o desenvolvimento da Hipertensão Renovascular. Rev Fac Ciênc Méd Sorocaba. 2014; 16(2):59-64.

10. Martelli A. Potencial da prática de exercícios físicos regulares como método não farmacológico no controle da Hipertensão Arterial Sistêmica. Desenvolvimento Pessoal. 2013; 3(2):19-51.

11. Pimenta E, Calhoun DA. Uso dos antagonistas da aldosterona no tratamento da hipertensão arterial refratária. Rev Bras Hipertens. 2007; 14(3): 180-1.

12. Marte AP, Santos RD. Bases fisiopatológicas da dislipidemia e hipertensão arterial. Rev Bras Hipertensão. 2008:14(4): 252-7.

13. Martelli A. Redução das concentrações de cloreto de sódio na alimentação visando a homeostase da pressão arterial. REGET/UFSM. 2014;18(1): 428-36

14. Sociedade Brasileira de Cardiologia; Sociedade Brasileira de Hipertensão; Sociedade Brasileira de Nefrologia. VI Diretrizes Brasileiras de Hipertensão, Arq. Brás. Cardiol. 2010;95(1 supl1):1-51.

15. Miranzi SSC, Ferreira FS, Iwamoto HH, Pereira GA, Miranzi MAS. Qualidade de vida de indivíduos com diabetes mellitus e hipertensão acompanhados por uma equipe de saúde da família. Texto contexto- enferm. 2008;17(4): 672-9.

16. Alavarce DC, Pierin AMG. Elaboração de uma hipermídia educacional para o ensino do procedimento de medida da pressão arterial. Rev Esc Enferm USP. 2011; 45(4):939-44.

17. Ribeiro AB. Atualização em hipertensão arterial: clínica, diagnóstico e terapêutica. São Paulo: Atheneu; 1996.

18. Simonetti JP, Batista L, Carvalho LR. Hábitos de saúde e fatores de risco em pacientes hipertensos. Rev. LatinoAm. Enfermagem. 2002;10(3):415-22.

19. Carvalho MV, Siqueira LB, Sousa ALL, Jardim PCBV. A Influência da Hipertensão Arterial na Qualidade de Vida Arq Bras Cardiol. 2013; 100(2):164-74.

20. Pinotti S, Mantovani MF, Giacomozzi LM. Percepção sobre a hipertensão arterial e qualidade de vida: contribuição para o cuidado de enfermagem. Cogitare Enferm. 2008;13(4): 526-34.

21. Carlotto MS, Camara SG. Preditores da Síndrome de Burnout em professores. Psicol Esc Educ. 2007; 11(1):101-10

22. Franco MEDP. Comunidade de conhecimento, pesquisa e formação do professor do ensino superior. Em M. C. Morosini. (Org.), Docência universitária e os desafios da realidade nacional. Brasília: Plano, 2001.
23. Santos MN, Marques AC. Condições de saúde, estilo de vida e características de trabalho de professores de uma cidade do sul do Brasil. Ciênc saúde coletiva. 2013; 18(3): 837-46

24. Moreira OC, Oliveira RAR, Andrade Neto F, Amorim W, Oliveira CEP, Doimo LA et al. Associação entre risco cardiovascular e hipertensão arterial em professores universitários. Rev Bras Educ Fís Esporte. 2011; 25(3): 397-406.

25. Martelli A, Zavarize SF. Fatores que influenciam a hipertensão arterial sistêmica e qualidade de vida em universitários do município de Mogi Guaçu - SP. Arch Health Invest . 2014; 3(5):32-42.

26. Costa RF. Composição corporal: teoria e prática da avaliação. São Paulo: Manole; 2003.

27. Woods SL, Froelicher ESS, Motzer SU. Enfermagem em cardiologia. 4. d. São Paulo: Manole; 2005.

28. Alves FG, Nakashima LMA, Klein GFS. Fatores de risco para hipertensão arterial sistêmica em docentes do curso de enfermagem de uma universidade privada da cidade de São Paulo. Saúde Coletiva. 2010; 7(42):179-82.

29. Baião LPM, Cunha RG. Doenças e/ou disfunções ocupacionais no meio docente: uma revisão de literatura. Rev Formação@Docente. 2013; 5(1):6-21.

30. Araujo TM, Godinho TM, Reis EJFB, Almeida MMG. Diferenciais de gênero no trabalho docente e repercussões sobre a saúde. Ciênc saúde coletiva. 2006; 11(4):1117-29.

31. Duarte CC; Prudente HA. O Impacto da Dupla Jornada dos Docentes de Ensino Superior na Geração do Estresse. RAF. 2016; 13(13): 44-63.

32. Batista MK, Grisci CLI, Gallon S, Figueiredo MD. Slow movement: trabalho e experimentação do tempo na vida líquido-moderna. Psicol Soc. 2013; 25(1):30-9.

33. Andrade PS, Cardoso TAO. Prazer e dor na docência: revisão bibliográfica sobre a Síndrome de Burnout. Saúde e Sociedade. 2012; 21(1):129-40.

34. Drager LF, Lorenzi-Filho G. Sono e pressão arterial: mecanismos fisiológicos de controle da pressão arterial no sono. Hipertensão. 2008; 11(3):84-9.

35. Mathias A, Sanchez RP, Andrade MMM. Incentivar hábitos de sono adequados: um desafio para os educadores. In: Pinho SZ, Saglietti JRC (eds.). Núcleos de Ensino da Unesp. São Paulo: UNESP; 2006. p.718-31.

36. Crispim CA, Zalcman I, Dáttilo M, Padilha HG, Tufik S Mello MT. Relação entre Sono e Obesidade: uma Revisão da Literatura. Arq Bras Endocrinol Metab 2007; 51(7):1041-9.

37. Nerbass FB, Andersen ML, Tufik S. Efeito da privação de sono no sistema cardiovascular. Rev Soc Cardiol Estado de São Paulo. 2010; 20(4):461-7.

38. Brown I.J, Tzoulaki I, Candeias V, Elliott P. Salt intakes around the world: implications for public health. Int J Epidemiol. 2009 ;38(3):791-813.

39. Salas CKTS, Spinelli MGN, Kawashima LM, Ueda AM. Teores de sódio e lipídios em refeições almoço consumidas por trabalhadores de uma empresa do município de Suzano, SP. Rev Nutr. 2009; 22(3):331-9.

40. Polonia J, Martins L. A comprehensive review on salt and health and current experience of worldwide salt reduction programmes. J Hum Hypertens. 2009; 23(11):771-2

41. V Diretrizes Brasileiras de Hipertensão Arterial. Arc Bras Cardiol. 2007; 89(3):e24-79 
42. Gonçalves S, Hardt JR, Silva ASS, Haas P. Hipertensão arterial e a importância da atividade física. Estud Biol. 2007; 29(67):205-13.

43. Bezerra IMP, Silva JC, Gomes SC. Mudanças nos aspectos sociais de hipertensos por meio de atividade física: desvelando a importância do profissional de educação física. Sau \& Transf Soc. 2011; 2(1): 56-64.

44. Halbert JA, Silagy CA, Finucane P, Withers RT, Hamdorf PA, Andrews GR. The effectiveness of exercise training in lowering blood pressure: a metaanalysis of randomized controlled trials of 4 weeks or longer. J Hum Hypertens. 1997; 11(10):641-9.

45. Oliveira RAR, Moreira OC, Andrade Neto F, Amorim W, Costa EG, Marins JCB. Prevalência de sobrepeso e obesidade em professores da Universidade Federal de Viçosa. Fisioter mov. 2011; 24(4):603-12.

46. Guyton AC, Hall JE. Tratado de fisiologia médica. Rio de Janeiro: Elsevie; 2011.

47. Barreto-Filho JAS, Krieger JE. Genética e hipertensão arterial: conhecimento aplicado à prática clínica. Rev Soc Cardiol Estado de São Paulo. 2003; 13(1):46-55.

48. Costa JSD, Barcellos FC, Sclowitz ML, Sclowitz IKT, Castanheira M, Olinto MTA et al. Prevalência de hipertensão arterial em adultos e fatores associados: um estudo de base populacional urbana em Pelotas, Rio Grande do Sul, Brasil. Arq Bras Cardiol. 2007; 88(1):59-65.

49. Silva JLL, Souza SL. Fatores de risco para hipertensão arterial sistêmica versus estilo de vida docente. Rev Eletrônica Enferm. 2004; 6(3):330-5.

\section{CONFLITO DE INTERESSES}

Os autores declaram não haver conflitos de interesse.

\section{AUTOR PARA CORRESPONDÊNCIA}

\section{Anderson Martelli}

martellibio@hotmail.com

Submetido em 14/06/2017

Aceito em 13/07/2017 